Erratum

\title{
Erratum to "Cancer Incidence and Mortality in a Cohort of US Blood Donors: A 20-Year Study"
}

\author{
Farnaz Vahidnia, ${ }^{1}$ Nora V. Hirschler, ${ }^{2}$ Maria Agapova, ${ }^{3}$ Artina Chinn, \\ Michael P. Busch, ${ }^{1,4}$ and Brian Custer ${ }^{1,4}$ \\ ${ }^{1}$ Blood Systems Research Institute, San Francisco, CA 94118, USA \\ ${ }^{2}$ Blood Centers of the Pacific, San Francisco, CA 94118, USA \\ ${ }^{3}$ University of Washington, Seattle, WA 98195, USA \\ ${ }^{4}$ Laboratory Medicine, University of California, San Francisco, CA 94143, USA
}

Correspondence should be addressed to Farnaz Vahidnia; fvahidnia@bloodsystems.org

Received 25 June 2014; Accepted 2 July 2014; Published 24 July 2014

Copyright (C) 2014 Farnaz Vahidnia et al. This is an open access article distributed under the Creative Commons Attribution License, which permits unrestricted use, distribution, and reproduction in any medium, provided the original work is properly cited.

In the original paper, there was an error in Figure 3. In the legend for Figure 3, donor and nondonor lines were reversed. Here, we provide Figure 3 with the correct legend. 


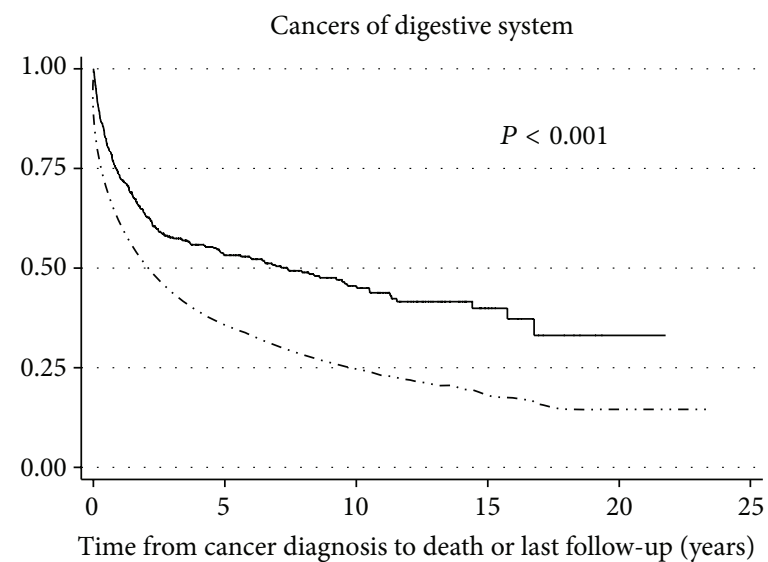

(a)

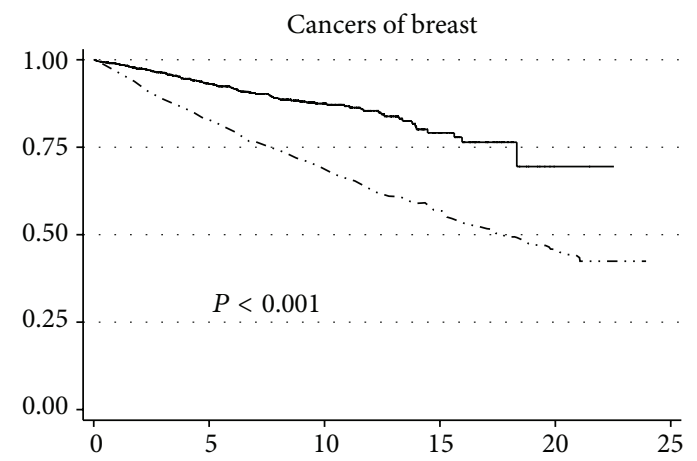

Time from cancer diagnosis to death or last follow-up (years)

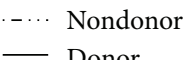

- Donor

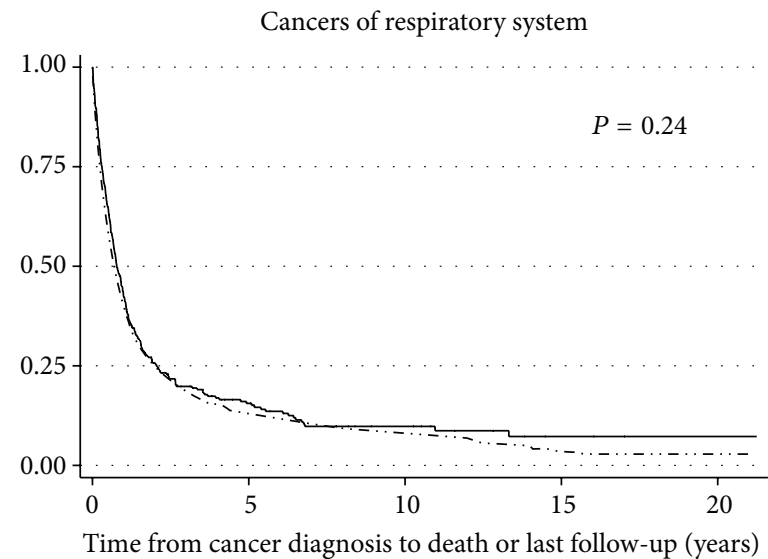

(b)

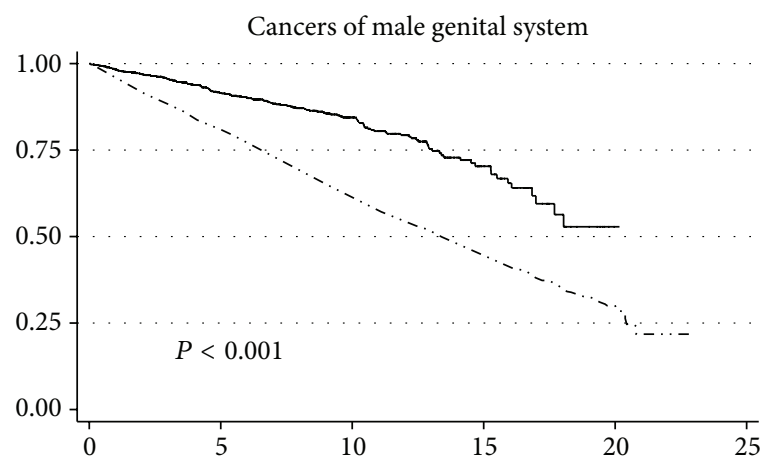

Time from cancer diagnosis to death or last follow-up (years)

-... Nondonor

Donor

(c)

(d)

FiguRE 3: KM survival curves for all-cause mortality for donors and nondonors with (a) cancers of digestive system $(P=0.24)$; $(\mathrm{b})$ cancers of respiratory system $(P<0.001)$; (c) breast cancers $(P<0.001)$; (d) cancers of male genital system $(P<0.001)$; $x$-axis represents survival time in years. 


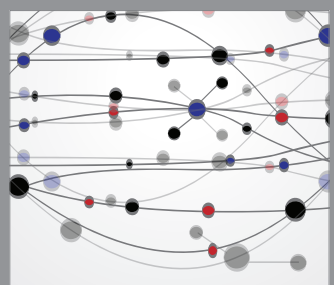

The Scientific World Journal
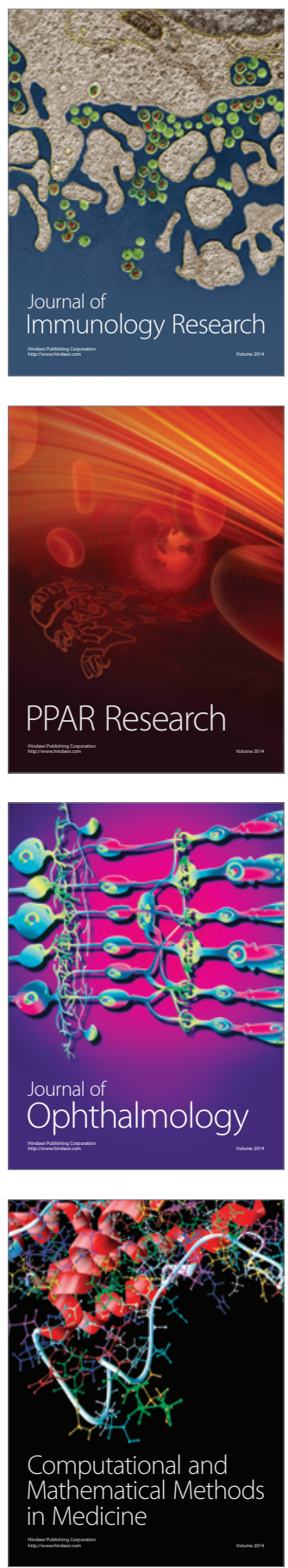

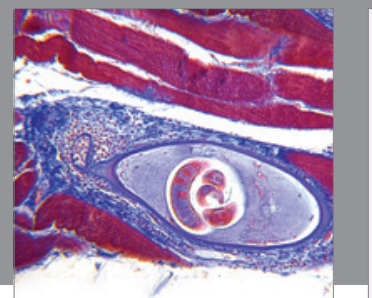

Gastroenterology

Research and Practice
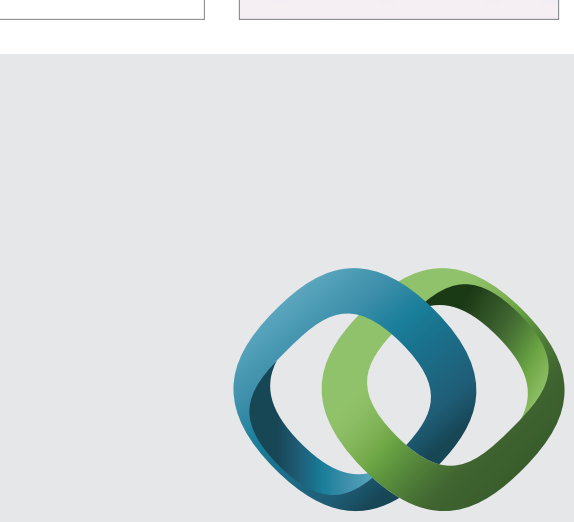

\section{Hindawi}

Submit your manuscripts at

http://www.hindawi.com
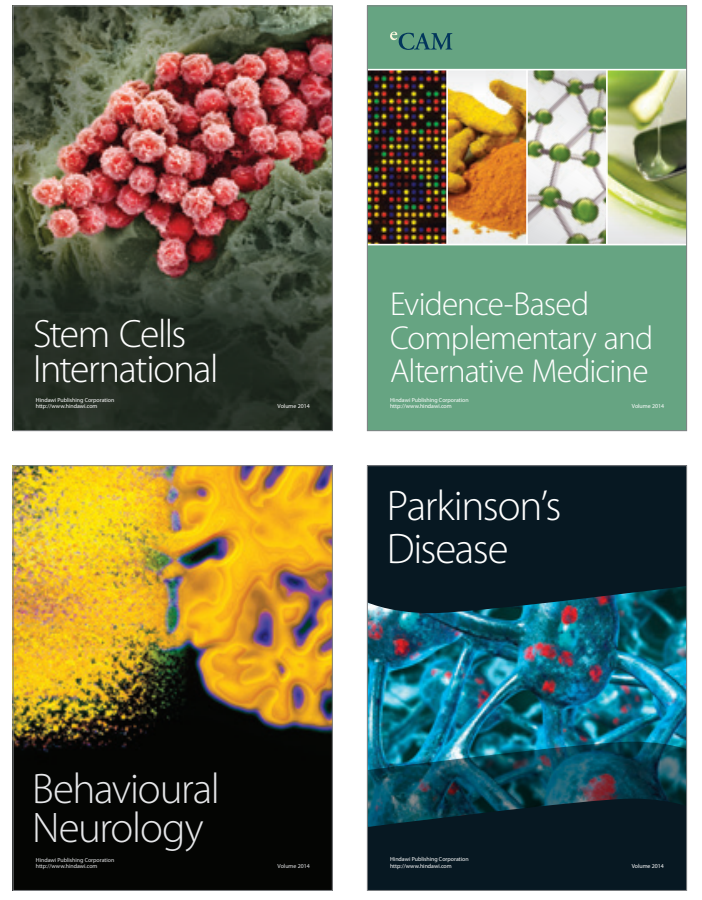
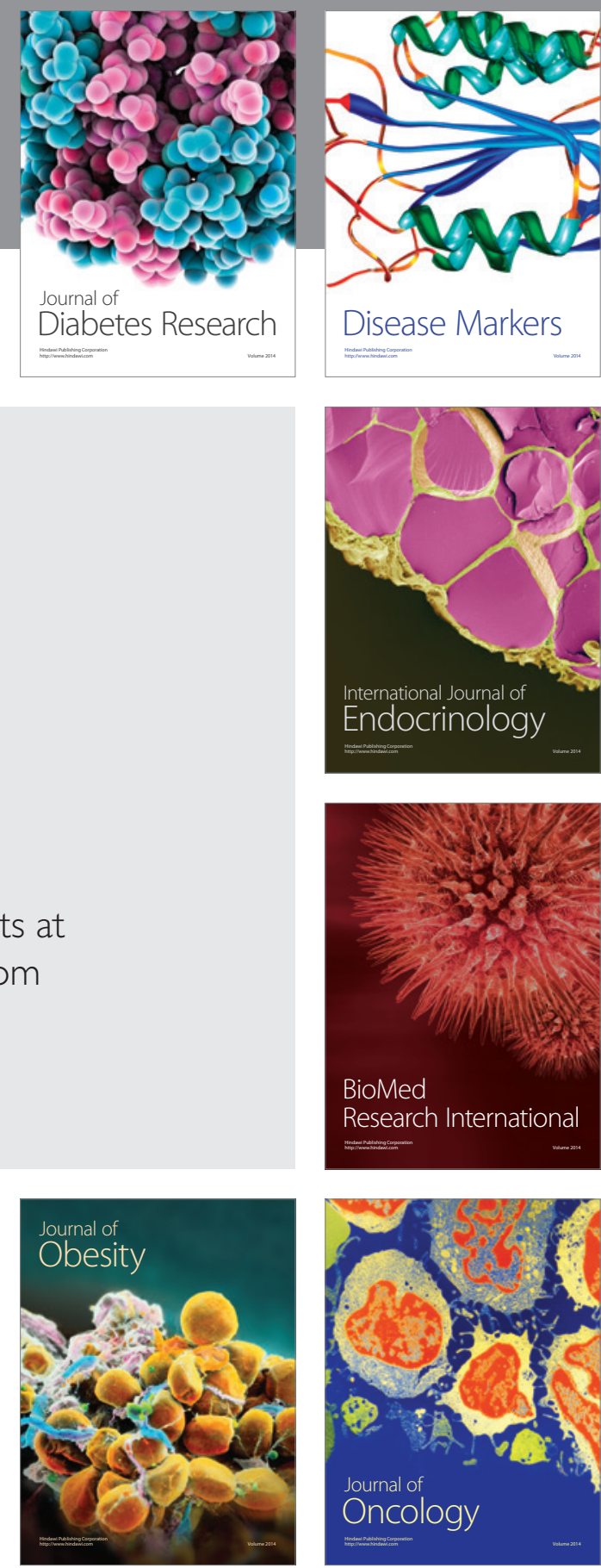

Disease Markers
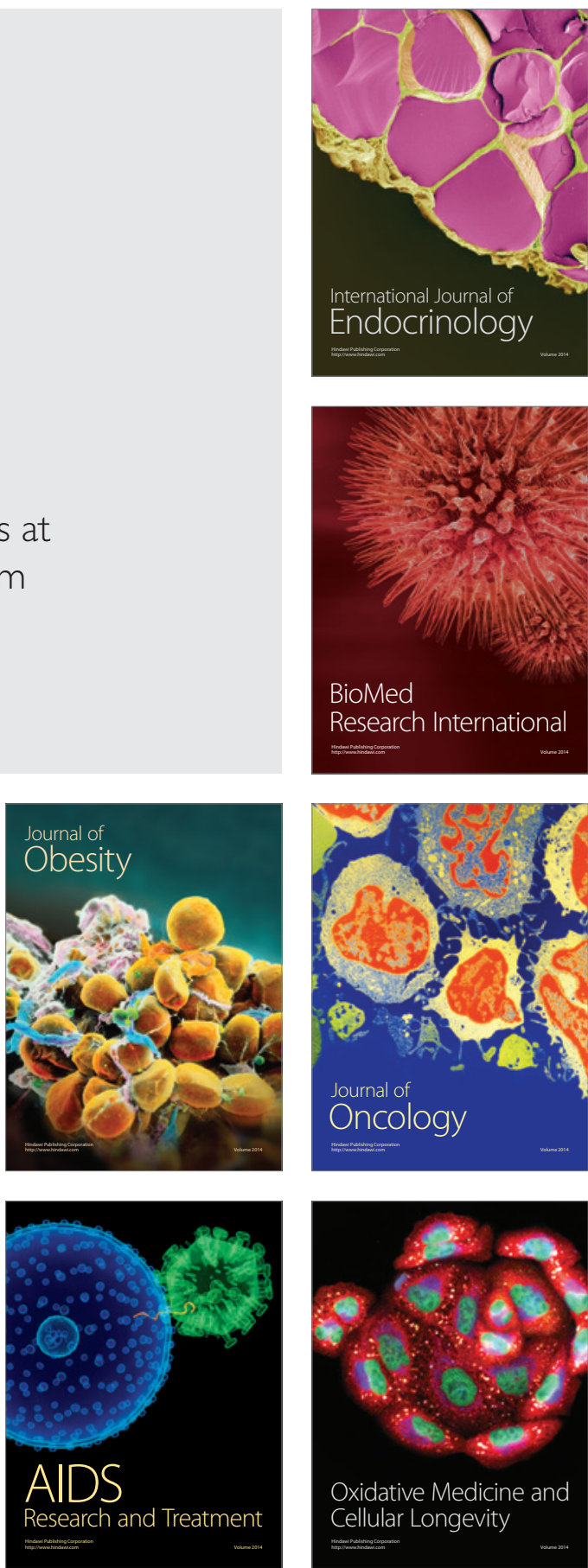